\title{
Electrospun gelatin/polycaprolactone nanofibrous membranes combined with a coculture of bone marrow stromal cells and chondrocytes for cartilage engineering
}

\author{
This article was published in the following Dove Press journal: \\ International Journal of Nanomedicine \\ 17 March 2015 \\ Number of times this article has been viewed
}

\author{
Xiaomin $\mathrm{He},{ }^{1, *}$ Bei Feng, 1,2,* \\ Chuanpei Huang,' \\ Hao Wang,' Yang Ge,' \\ Renjie Hu,' Meng Yin,' \\ Zhiwei Xu,' Wei Wang,' \\ Wei Fu, ',2 Jinghao Zheng' \\ 'Department of Pediatric \\ Cardiothoracic Surgery, ${ }^{2}$ Institute \\ of Pediatric Translational Medicine, \\ Shanghai Children's Medical Center \\ School of Medicine, Shanghai Jiao Tong \\ University, Shanghai, People's Republic \\ of China
}

*These authors contributed equally to this work

Correspondence: Wei Fu Department of Pediatric Cardiothoracic Surgery, Institute of Pediatric Translational Medicine, Shanghai Children's Medical Center, School of Medicine, Shanghai Jiao Tong University, 1678 Dong Fang Road, Shanghai 200127, People's Republic of China

Tel +862138625553

Fax +86 2I 50904612

Email fuweizhulu@।63.com

Jinghao Zheng

Department of Pediatric Cardiothoracic Surgery, Shanghai Children's Medical Center School of Medicine, Shanghai Jiao Tong University, 1678 Dong

Fang Road, Shanghai 200 I27,

People's Republic of China

Tel +86 2l 38625553

Fax +86 2I 50904612

Email zhengjh210@163.com
Abstract: Electrospinning has recently received considerable attention, showing notable potential as a novel method of scaffold fabrication for cartilage engineering. The aim of this study was to use a coculture strategy of chondrocytes combined with electrospun gelatin/ polycaprolactone (GT/PCL) membranes, instead of pure chondrocytes, to evaluate the formation of cartilaginous tissue. We prepared the GT/PCL membranes, seeded bone marrow stromal cell (BMSC)/chondrocyte cocultures (75\% BMSCs and 25\% chondrocytes) in a sandwich model in vitro, and then implanted the constructs subcutaneously into nude mice for 12 weeks. Gross observation, histological and immunohistological evaluation, glycosaminoglycan analyses, Young's modulus measurement, and immunofluorescence staining were performed postimplantation. We found that the coculture group formed mature cartilage-like tissue, with no statistically significant difference from the chondrocyte group, and labeled BMSCs could differentiate into chondrocyte-like cells under the chondrogenic niche of chondrocytes. This entire strategy indicates that GT/PCL membranes are also a suitable scaffold for stem cell-based cartilage engineering and may provide a potentially clinically feasible approach for cartilage repairs.

Keywords: electrospinning, nanocomposite, cartilage tissue engineering, nanomaterials, stem cells

\section{Introduction}

Owing to the limited self-repair capacity of cartilage because of its poor regenerative ability and deficient blood supply, cartilage tissue engineering provides a promising approach for cartilage regeneration in patients with various cartilage defects. ${ }^{1-5}$ In recent years, considerable attention has been given to biodegradable synthetic polymers and their application in cartilage tissue engineering. ${ }^{6,7}$ Scaffolds seeded with chondrocytes or stem cells have been widely investigated for use in the tissue engineering of cartilage. However, most of the scaffolds investigated were not able to form ideal cartilage tissue, restricting their further clinical application. Therefore, the selection of appropriate scaffolds is critical for the engineering of effective cartilage.

Recently, electrospinning has received considerable attention as an alternative approach for the fabrication of unique scaffolds. ${ }^{8,9}$ Electrospinning can produce polymer fibers with diameters down to nanoscale dimensions, which could structurally mimic the natural extracellular matrix (ECM). ${ }^{10,11}$ To date, a range of polymers, including polyglycolic acid, polycaprolactone (PCL), polylactic acid, and their copolymers, have been developed as scaffolds by electrospinning for tissue engineering applications. ${ }^{12-15}$ 
Furthermore, blending synthetic polymers and natural materials by electrospinning could combine the advantages of both synthetic and natural materials, potentially improving cell affinity while offering ideal mechanical properties for tissue engineering applications. ${ }^{16-18}$

In our previous study, we fabricated electrospun gelatin/ polycaprolactone (GT/PCL) nanofibrous membranes, and found that acetic acid could improve the miscibility, contributing to obtaining finer and compositionally homogeneous hybrid nanofibers. ${ }^{19}$ Furthermore, GT/PCL membranes seeded with chondrocytes in a sandwich model could form good-quality cartilage with precise three-dimensional (3-D) structures. ${ }^{20}$ However, chondrocytes as seed cells have limited cell sources and poor capability to expand in vitro. Besides, chondrocytes are prone to dedifferentiation after monolayer expansion, affecting the quality of the neocartilage. ${ }^{21}$ Given the limitations of chondrocytes, it is necessary to consider other attractive alternative cell sources for seeding on to the GT/PCL membranes for engineering cartilage.

Bone marrow stromal cells (BMSCs) have a self-renewal capacity, long-term viability, and a potential for multilineage differentiation, such as osteogenic, chondrogenic, adipogenic, and myogenic lineages. ${ }^{22,23}$ Over the past decade, BMSCs have been widely used for cartilage tissue engineering. ${ }^{24,25}$ However, the chondrogenic induction of BMSCs by common in vitro protocols for engineering cartilage tended to differentiate toward the hypertrophic phenotype and resulted in calcification, especially at heterotopic sites. ${ }^{26}$ The recent coculture strategy, combining the chondrogenic niche of chondrocytes with the proliferative potential of BMSCs, could enhance BMSC chondrogenesis and prevent hypertrophy, resulting in stable cartilage formation. ${ }^{27,28}$ In the coculture system, paracrine-soluble chondrogenic factors, including transforming growth factor $\beta 1$ (TGF- $\beta 1$ ), insulinlike growth factor 1 (IGF-1), bone morphogenetic protein 2 (BMP-2), and parathyroid hormone-related protein (PTHrP) released by chondrocytes, provided potent chondroinductive signals and improved chondrogenesis, and suppressed the hypertrophic development of BMSCs. ${ }^{29,30}$ These findings indicate that the coculture of BMSCs and chondrocytes is economical, easy, and promising for the generation of stable tissue-engineered cartilage. However, it remains unclear whether GT/PCL membranes combining a coculture of BMSCs and chondrocytes could successfully generate neocartilage in the sandwich model.

In this study, we used a coculture strategy instead of pure chondrocytes to engineer cell-scaffold constructs using GT/PCL membranes seeded with BMSC/chondrocyte cocultures in the sandwich model in vitro and then implanted the constructs subcutaneously into nude mice for 12 weeks to further investigate whether GT/PCL membranes could be suitable for stem cell-based cartilage engineering. Moreover, BMSCs were labeled with CM-Dil before construction in vitro to provide scientific evidence that BMSCs could undergo chondrogenic differentiation in vivo and form stable cartilage.

\section{Materials and methods Experimental animals}

Nine male New Zealand white rabbits ( 8 weeks old) were purchased from Shanghai Chedun Experimental Animal Raising Farm (Shanghai, People's Republic of China). Eighteen male nude mice (7 weeks old) were purchased from Shanghai Slaccas Experimental Animal Ltd. (Shanghai, People's Republic of China). All experimental protocols were approved by the Animal Care and Experiment Committee of Shanghai Jiao Tong University School of Medicine.

\section{Fabrication of GT/PCL membranes by electrospinning}

GT/PCL membranes were fabricated by electrospinning, as previously described. ${ }^{19,20}$ In brief, polymers of gelatin type A (300 Bloom) from porcine skin in powder form and PCL (MW, 80,000) were obtained from Sigma-Aldrich (St Louis, MO, USA). Then, gelatin and PCL were dissolved separately in trifluoroethanol (TFE) at a concentration of $10 \mathrm{wt} \%$ and stirred vigorously at room temperature for 24 hours. Before electrospinning, the two solutions were mixed in a 50:50 (v:v) ratio with a trace amount of acetic acid (acetic acid/TFE $0.2 \%$ ) by stirring at room temperature for 0.5 hours. The electrospinning conditions were as follows: $2.0 \mathrm{~mL} / \mathrm{h}$ injection rate, $10 \mathrm{kV}$ voltage, and $12 \mathrm{~cm}$ distance between the syringe nozzle and the grounded aluminum sheet $\left(200 \times 200 \mathrm{~mm}^{2}\right)$. The GT/PCL membranes were vacuum dried for 1 week at room temperature. The membranes were then tailored into a round shape (shown in Figure 1A) and lyophilized in a vacuum freeze-drier (Freezone 2.5L Triad; Labconco, Kansas City, MO, USA) for 24 hours. Eventually, the GT/PCL membranes were sterilized for 0.5 hours under ultraviolet (UV) irradiation for subsequent uses.

\section{Isolation and culture of cells}

BMSCs and chondrocytes from New Zealand white rabbits were isolated and cultured, respectively, according to previously established methods. ${ }^{18,31}$ Briefly, fresh bone marrow was harvested, centrifuged, washed, and then resuspended onto culture dishes at a cell density of $5 \times 10^{5}$ nucleated 
A

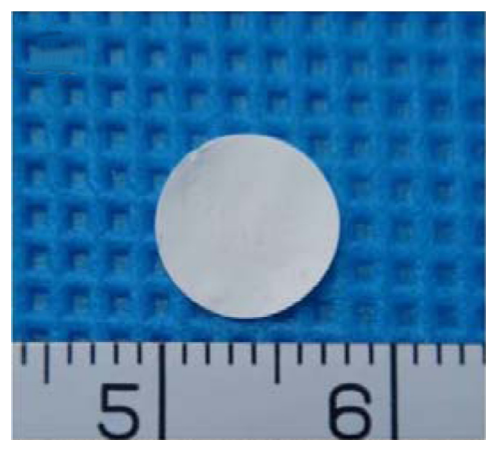

B

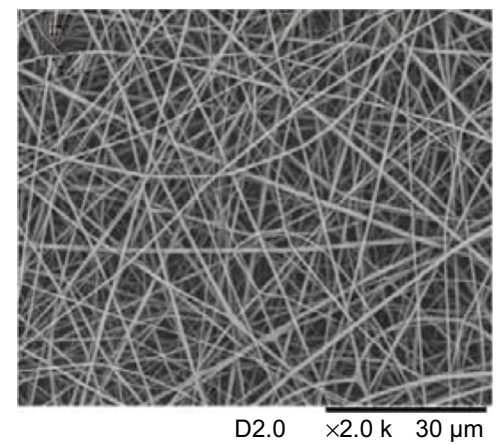

C

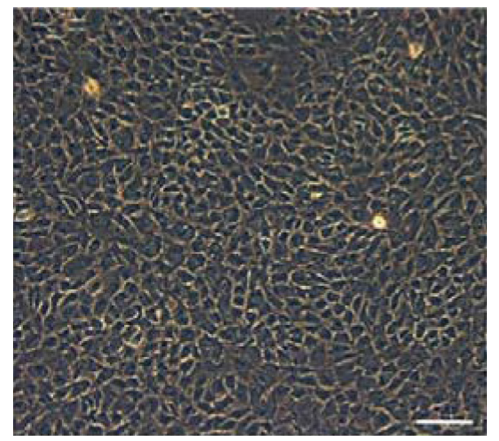

D

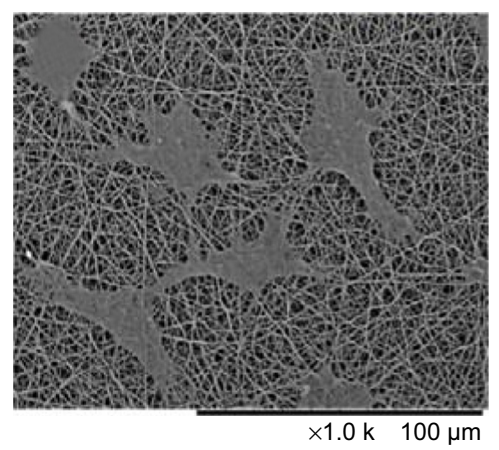

$\mathbf{E}$

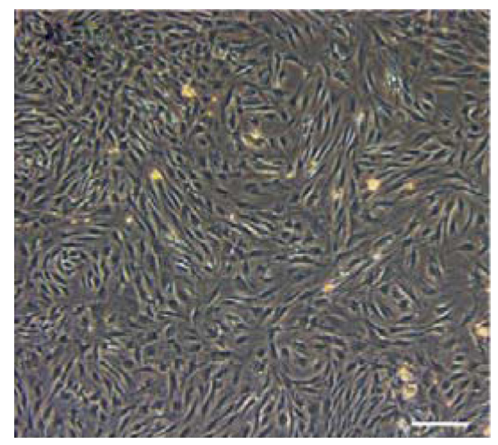

$\mathbf{F}$

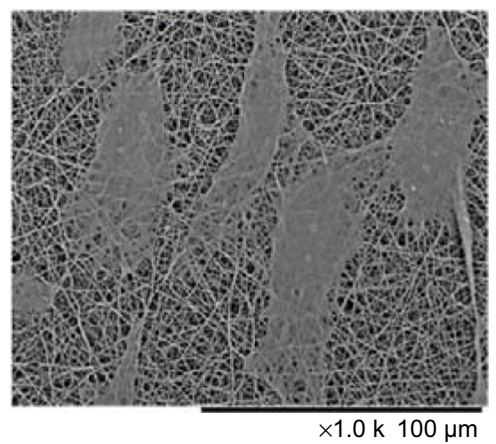

Figure I Characterization and biocompatibility of GT/PCL nanofibrous membrane.

Notes: (A) Gross view of the sheet of electrospun GT/PCL nanofibrous membrane. (B) SEM image of GT/PCL membrane. (C) Morphology of Passage I chondrocytes using a light microscope. Scale bar: $200 \mu \mathrm{m}$. (D) Morphology of chondrocytes on GT/PCL membranes after 2 days of cell culture in vitro. (E) Morphology of Passage 2 BMSCs using a light microscope. Scale bar: $200 \mu \mathrm{m}$. (F) Morphology of BMSCs on GT/PCL membranes after 2 days of cell culture in vitro.

Abbreviations: GT/PCL, gelatin/polycaprolactone; SEM, scanning electron microscopic; BMSC, bone marrow stromal cell.

cells $/ \mathrm{cm}^{2}$ in Dulbecco's Modified Eagle's Medium (DMEM; Hyclone, Logan, UT, USA) containing 10\% fetal bovine serum (FBS; Bioind, Beit HaEmek, Israel), $100 \mathrm{U} / \mathrm{mL}$ penicillin, and $100 \mathrm{U} / \mathrm{mL}$ streptomycin (Hyclone). When primary cells reached over $80 \%-90 \%$ confluence, the cells were digested with $0.25 \%$ trypsin plus $0.02 \%$ Ethylene Diamine Tetraacetie Acid (EDTA) (Gibco, Waltham, MA, USA) and then subcultured at a cell density of $2 \times 10^{4}$ cells $/ \mathrm{cm}^{2}$. Passage 2 BMSCs were used for further experiments.

Fresh auricular cartilages were harvested, washed, and digested with $0.25 \%$ trypsin plus $0.02 \%$ EDTA at $37^{\circ} \mathrm{C}$ for 30 minutes, minced into approximately $1 \mathrm{~mm}^{3}$ pieces, and then digested with $0.1 \%$ collagenase NB4 (SERVA, Heidelberg, Germany) in serum-free DMEM at $37^{\circ} \mathrm{C}$ for about 12-16 hours. Primary chondrocytes were harvested, counted, and seeded onto culture dishes at a cell density of $2 \times 10^{4} \mathrm{cells} / \mathrm{cm}^{2}$ in regular medium. Passage 1 chondrocytes were used for further experiments.

\section{Cell adhesion and growth on GT/PCL membranes}

To detect the cytocompatibility of GT/PCL membranes, the morphology and adhesion of BMSCs and chondrocytes on GT/PCL membranes were observed by scanning electron microscopy (SEM). The BMSCs or chondrocytes were seeded onto GT/PCL membranes at a density of $2 \times 10^{4}$ cells/ well in 24-well plates ( $2 \mathrm{~cm}^{2}$ per well). Two days after cell seeding, samples were fixed with $2.5 \%$ glutaraldehyde overnight at $4{ }^{\circ} \mathrm{C}$. Samples were rinsed and dehydrated with graded concentrations $(50 \%, 70 \%, 80 \%, 90 \%, 95 \%$, and $100 \% \mathrm{v} / \mathrm{v}$ ) of ethanol. Subsequently, the samples were critical-point dried and examined by SEM.

\section{Engineering of cell-scaffold constructs}

BMSCs and chondrocytes were collected and mixed at a ratio of 3:1 (BMSCs:chondrocytes) with an ultimate concentration of $1 \times 10^{8}$ cells $/ \mathrm{mL}$. Then, the cell-scaffold constructs (coculture group) were engineered using the sandwich model, as described previously. ${ }^{32}$ Briefly, one GT/PCL membrane was placed in the bottom of a well of 6-well plates seeded with $5 \mu \mathrm{L}$ of the mixed cell suspension. Another sheet was then stacked on top of the first sheet using sterile forceps, followed by seeding with the same number of cells. The procedure was repeated until ten sheets were stacked. Pure chondrocytes or BMSCs were likewise constructed with GT/PCL membranes as a positive control (chondrocyte group) and negative control (BMSC group). All cell-scaffold constructs were then cultured in regular medium in vitro for 
1 week and then implanted subcutaneously into nude mice for 12 weeks ( $n=5$ for each group).

\section{Gross observation}

After implantation for 12 weeks, the mice were sacrificed, and the cell-scaffold constructs were dissected and harvested from the aforementioned three groups for further investigation. Each construct was observed grossly for shape, color, and size, and palpated with forceps to assess mechanical rigidity. Furthermore, the thickness and wet weight of each construct were measured and subjected to statistical analysis.

\section{Quantification of glycosaminoglycan content}

Glycosaminoglycan (GAG) analysis was performed according to methods previously described. ${ }^{33}$ Samples from each group were digested in papain solution (Sigma-Aldrich). The GAG content was quantified by adding the sample to 1,9-dimethylmethylene blue (Sigma-Aldrich) dye solution and measuring the absorbance value at $520 \mathrm{~nm}$ using shark chondroitin sulfate (Sigma-Aldrich) as the standard. The rabbit auricular cartilages were used as a normal control.

\section{Biomechanical analysis}

The mechanical properties of cell-scaffold constructs from each group were measured using a biomechanical testing machine (Instron-5542; Instron Corporation, Norwood, MA, USA), as previously described. ${ }^{34}$ Samples were prepared and a constant compressive strain rate of $0.5 \mathrm{~mm} / \mathrm{min}$ was applied until $80 \%$ of maximal deformation was achieved, and a stress-strain curve was then generated. The Young's modulus of the samples were calculated based on the slope of the stress-strain curve. The rabbit auricular cartilages were used as a normal control.

\section{Histological and immunohistochemical staining}

After gross examination, the samples harvested from all the groups were subjected to histological and immunohistochemical examinations as previously described. ${ }^{32}$ The samples were fixed in $4 \%$ paraformaldehyde, embedded in paraffin, and then sectioned into $5 \mu \mathrm{m}$ sections. The sections were stained with hematoxylin and eosin (H\&E) to assess tissue structure and Toluidine blue and Safranin O to locate the GAG deposits. Expression of type II collagen was detected with a mouse antihuman type II collagen monoclonal antibody (1:200; Abcam, Cambridge, UK), followed by a secondary antibody (1:50; Dako, Glostrup, Denmark) in phosphate-buffered saline (PBS), and diaminobenzidine tetrahydrochloride (Dako) was used for color development.

\section{Labeling and tracing chondrogenesis of BMSCs}

To trace the differentiation of BMSCs in cell-scaffold constructs implanted subcutaneously into nude mice, the fluorescent carbocyanine CM-Dil (Molecular probes, Waltham, MA, USA) was used to label the BMSC membranes, similar to a previous report. ${ }^{35}$ In brief, BMSCs were collected, counted, and then labeled with CM-Dil $(1: 100,10 \mu \mathrm{L} / \mathrm{mL})$ for 30 minutes at $37^{\circ} \mathrm{C}$. Then, the labeled BMSCs were washed with PBS three times and then mixed with chondrocytes at a ratio of $3: 1$ (BMSCs:chondrocytes) at an ultimate concentration of $1 \times 10^{8}$ cells/mL. Next, the mixed cells were seeded onto GT/PCL membranes in the sandwich model. The cell-scaffold constructs were also cultured in regular medium in vitro for 1 week and then implanted subcutaneously into nude mice for 12 weeks.

After 12 weeks of implantation, the labeled BMSCs were analyzed by immunofluorescence staining for cartilage marker type II collagen. Seven-micrometer frozen sections of samples were prepared, fixed with 4\% paraformaldehyde for 2 hours at room temperature, and washed with PBS, then $0.5 \%$ Triton X-100 (Sigma-Aldrich) for 30 minutes, again washed with PBS, blocked with 10\% donkey serum (Multisciences Biotech, Hangzhou, People's Republic of China) in PBS for 30 minutes at $37^{\circ} \mathrm{C}$, and then incubated for 2 hours at $37^{\circ} \mathrm{C}$ with a mouse antihuman type II collagen monoclonal antibody (1:200). After rinsing with PBS, the samples were subsequently incubated with Alexa Fluor 488-conjugated donkey secondary antibody (Life Technologies, Waltham, MA, USA) for $30 \mathrm{~min}$ utes at $37^{\circ} \mathrm{C}$. Nuclei were counterstained with $4^{\prime}, 6$-diamidino2-phenylindole (DAPI) (1:500; Enzo Life Sciences, Lausen, Switzerland) for 30 seconds. Immunostaining was observed and analyzed using an inverted fluorescence microscope (Leica DMI3000B; Leica Microsystems, Wetzlar, Germany).

\section{Statistical analysis}

All quantitative data are presented as mean \pm standard deviation (SD). The different groups were compared using singlefactor analysis of variance (ANOVA). A value of $P<0.05$ was considered statistically significant.

\section{Results \\ Characterization and cytocompatibility of GT/PCL membranes}

Electrospun GT/PCL membranes with a 50:50 gelatin-toPCL ratio were cut into $8 \mathrm{~mm}$ round sheets with an average 
thickness of $24 \mu \mathrm{m}$ (Figure 1A). Figure 1B shows the morphology of GT/PCL membranes as visualized by SEM. The membranes were found to be randomly uniform, beadfree, smooth structures with an average nanofiber diameter of $434 \pm 130 \mathrm{~nm}$. To evaluate the cytocompatibility of GT/PCL membranes, BMSCs and chondrocytes were seeded and cultured on the GT/PCL membranes, respectively. After 2 days of culture, both cells could adhere, spread, and proliferate on the surface of the GT/PCL membranes with their original shapes, according to SEM images (Figure 1).

\section{Gross observation}

The cell-scaffold constructs were built using the sandwich model with ten layers. The cells were closely adhered to the GT/PCL membranes with a regular cylindrical shape (Figure 2A). After 1 week of culture in vitro, the cellscaffold constructs were implanted subcutaneously into nude mice (Figure 2B). After 12 weeks of implantation, all constructs were harvested from nude mice (Figure 2C). Gross observation showed that all postimplantation constructs almost retained their original rounded shapes. As compared with the constructs in the chondrocyte group, those in the coculture group also formed cartilage-like tissue with an ivory-white appearance and good flexibility (Figure 2D and E). In contrast, the constructs in the BMSC group showed a reddish appearance without any luster or flexibility (Figure 2F).

\section{Quantitative analysis of thickness, wet weight, GAG content, and Young's modulus}

The thicknesses and wet weights of the cell-scaffold constructs were measured in all groups. There was no statistically significant difference in thickness or wet weight between the chondrocyte group and the coculture group (Figure $3 \mathrm{~A}$ and $\mathrm{B}$, $P>0.05)$. However, the constructs in the BMSC group were significantly lower in thickness and wet weight compared with those in the chondrocyte and coculture groups (Figure $3 \mathrm{~A}$ and $\mathrm{B}, P<0.01$ ), indicating that the pure BMSCs had not promoted the formation of cartilage, since the thickness and wet weight of the constructs had not increased in the BMSC group.

The GAG contents of the constructs from each group were also analyzed (Figure 3C). Normalized to wet weight, the GAG contents were significantly increased in both the chondrocyte group and the coculture group, with no statistically significant difference $(P>0.05)$. In contrast, the pure BMSCs had secreted almost no cartilage ECM. The GAG content was very low in the BMSC group, and significantly lower than that in the chondrocyte or coculture group $(P<0.01)$.
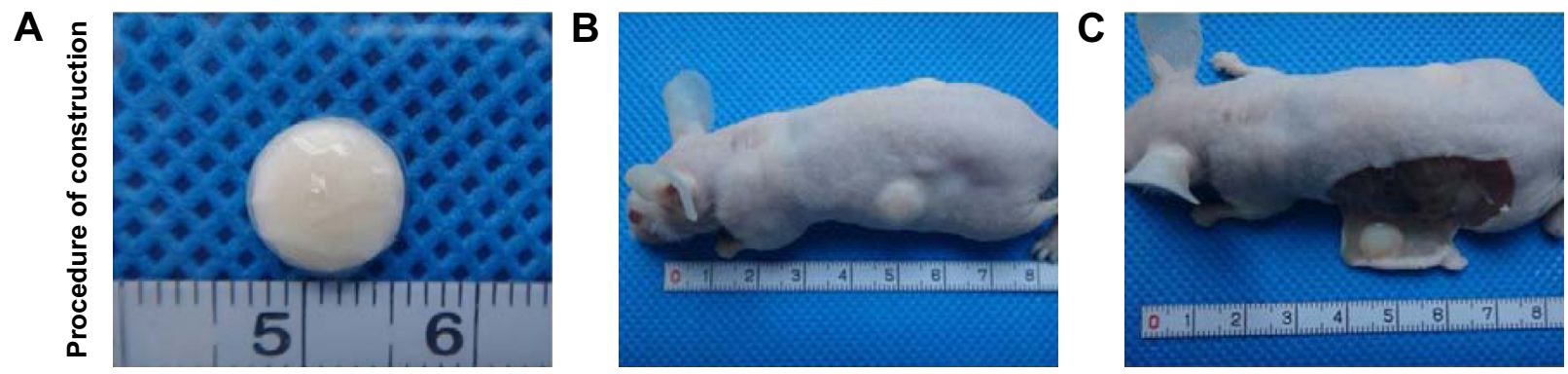

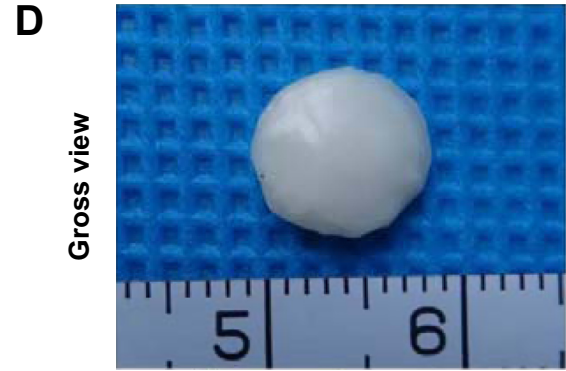

Chondrocytes group

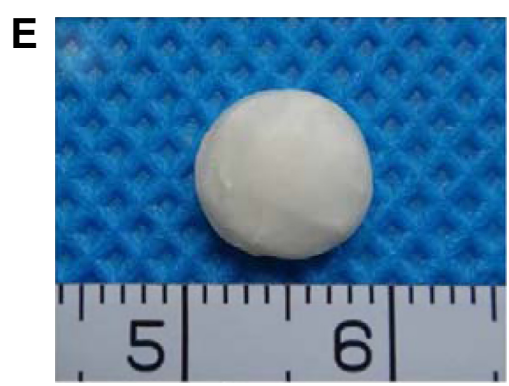

Coculture group

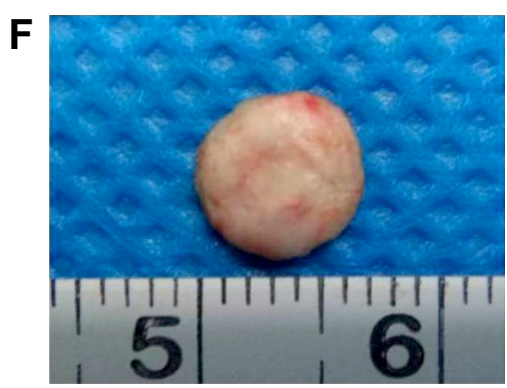

BMSCs group

Figure 2 Engineering cell-scaffold constructs in vitro and the formation of cartilage in vivo.

Notes: (A) Gross view of cell-scaffold constructs engineered in the sandwich model in vitro. (B) Cell-scaffold constructs were implanted subcutaneously into nude mice after being cultured for I week in vitro. (C) Gross view of cell-scaffold constructs 12 weeks postimplantation. (D-F) Gross view of constructs I 2 weeks postimplantation in the chondrocyte group, coculture group, and BMSC group, respectively. Minimum scale: $1 \mathrm{~mm}$.

Abbreviation: BMSC, bone marrow stromal cell. 
A

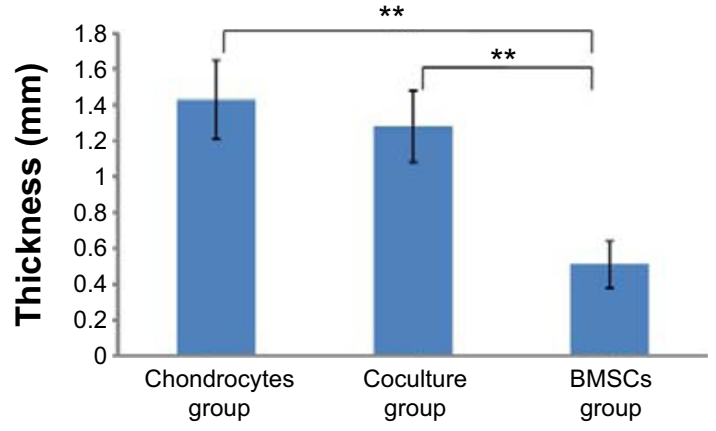

C

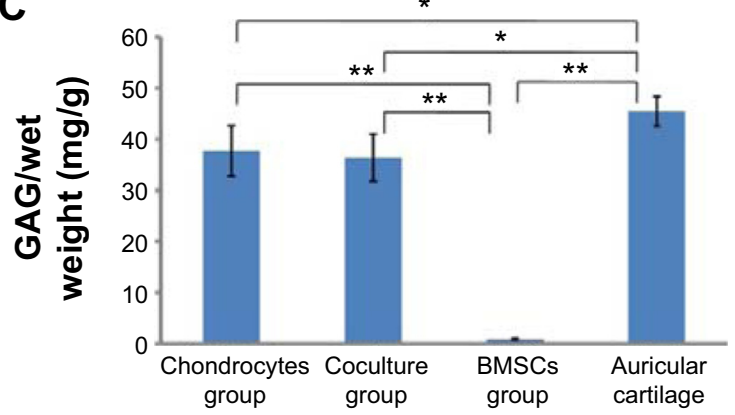

B

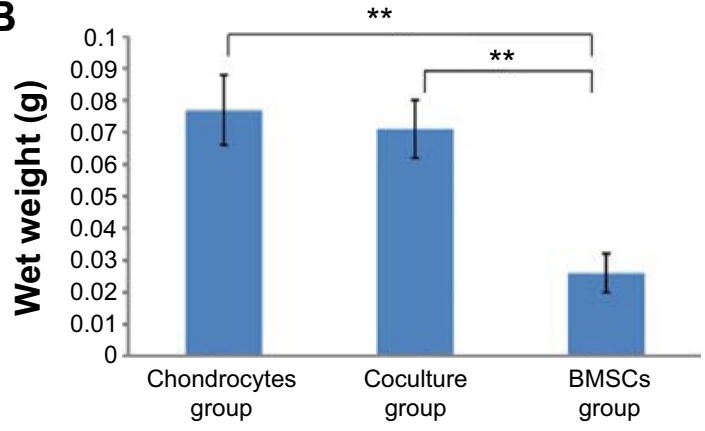

D

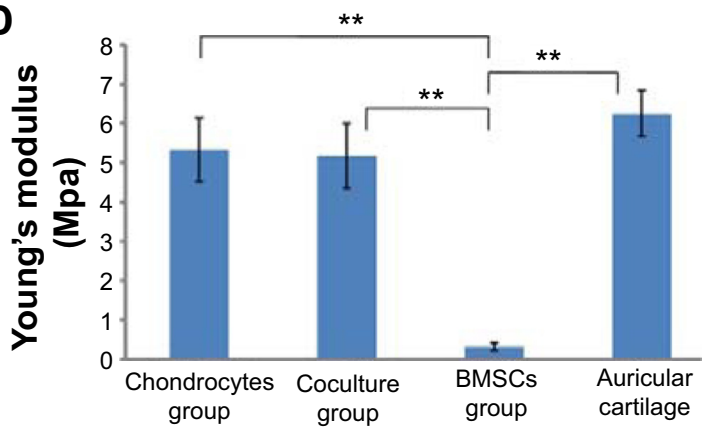

Figure 3 Quantitative analysis of thickness, wet weight, GAG content, and Young's modulus.

Notes: (A) Average thicknesses of cell-scaffold constructs in the chondrocyte group, coculture group, and BMSC group, respectively. (B) Average wet weights of cell-scaffold constructs in the different groups. (C) GAG content normalized to wet weight for cell-scaffold constructs in the different groups. Rabbit auricular cartilages were used as a normal control. (D) Young's modulus of cell-scaffold constructs in the different groups. The rabbit auricular cartilages were used as a normal control. Mean \pm SD ( $\mathrm{n}=4$ ); $* P<0.05, * * P<0.01$.

Abbreviations: GAG, glycosaminoglycan; BMSC, bone marrow stromal cell; SD, standard deviation.

The mechanical properties of the constructs from each group were evaluated using a static mechanical spectrometer (Figure 3D). The BMSC group displayed almost no mechanical properties. However, the Young's modulus of the constructs was significantly higher in both the chondrocyte group and the coculture group, without statistically significant difference $(P>0.05)$. This indicates that the BMSCs in the coculture group played an equal role as compared with the chondrocyte group.

\section{Histological and immunohistological observations}

Histological staining could further support the aforementioned observations in terms of cartilage formation. Mature cartilages with a large number of typical lacunar structures were observed between each layer of the constructs in both the chondrocyte group and the coculture group. Moreover, almost no central areas of staining defects were observed in these constructs. In contrast, almost no tissue structures existed among the layers of the constructs in the BMSC group. However, the undegraded GT/PCL membranes could be observed in all groups (Figure 4).

Toluidine blue and Safranin O can locate GAG deposits in the cartilage tissue. Type II collagen is the main component of the ECM in natural cartilage. As shown in Figure 5, Toluidine blue- and Safranin O-positive staining in both the chondrocyte group and the coculture group indicated that abundant, homogeneous cartilage ECM accumulated and was distributed within the constructs after implantation. Immunohistochemistry staining further showed that homogeneous extracellular staining for type II collagen was significantly increased in both the chondrocyte group and the coculture group. However, the constructs showed no GAG or type II collagen depositions in the BMSC group. These results show that the coculture group could also successfully form abundant mature cartilage as compared with the chondrocyte group.

\section{Tracing chondrogenesis of labeled BMSCs in vivo}

To further confirm the direct chondrogenesis of BMSCs induced by chondrocytes during the coculture system, CM-Dillabeled BMSCs were mixed with chondrocytes in a 3:1 ratio to form engineered cartilage by seeding within the GT/PCL membranes in the same sandwich model in vitro (Figure 6A), and the constructs were implanted in vivo for 12 weeks. CM-Dil-labeled BMSCs presented a red appearance, resulting 

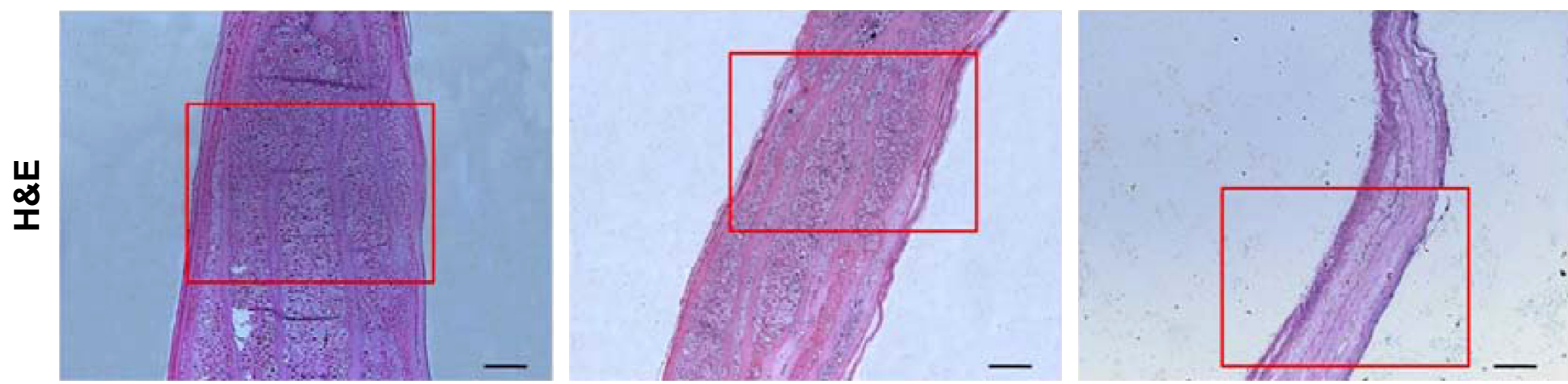

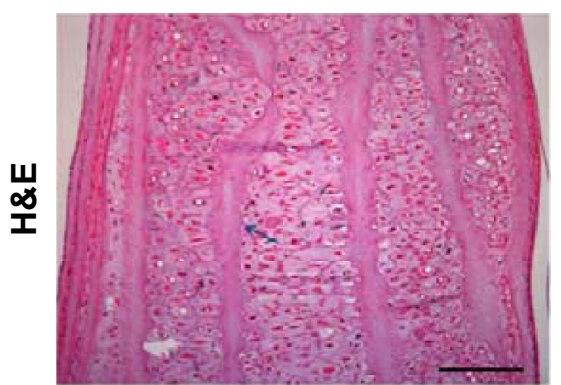

Chondrocytes group

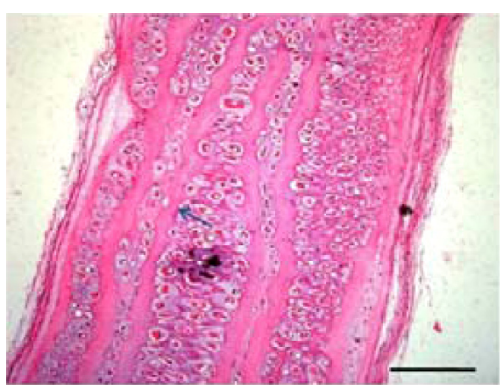

Coculture group

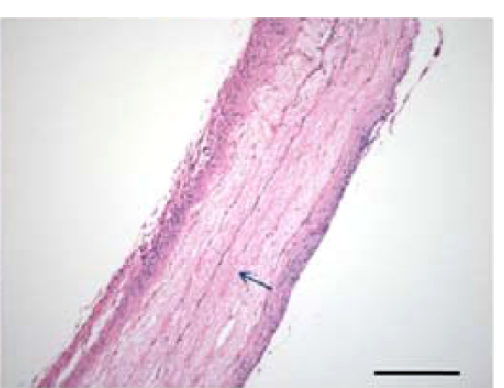

BMSCs group

Figure 4 Histology of cell-scaffold constructs after 12 weeks in vivo in the different groups.

Notes: Tissue sections were stained with H\&E. Arrows show nondegraded GT/PCL nanofibrous membranes. Scale bars: $200 \mu \mathrm{m}$.

Abbreviations: H\&E, hematoxylin and eosin; BMSC, bone marrow stromal cell; GT/PCL, gelatin/polycaprolactone.
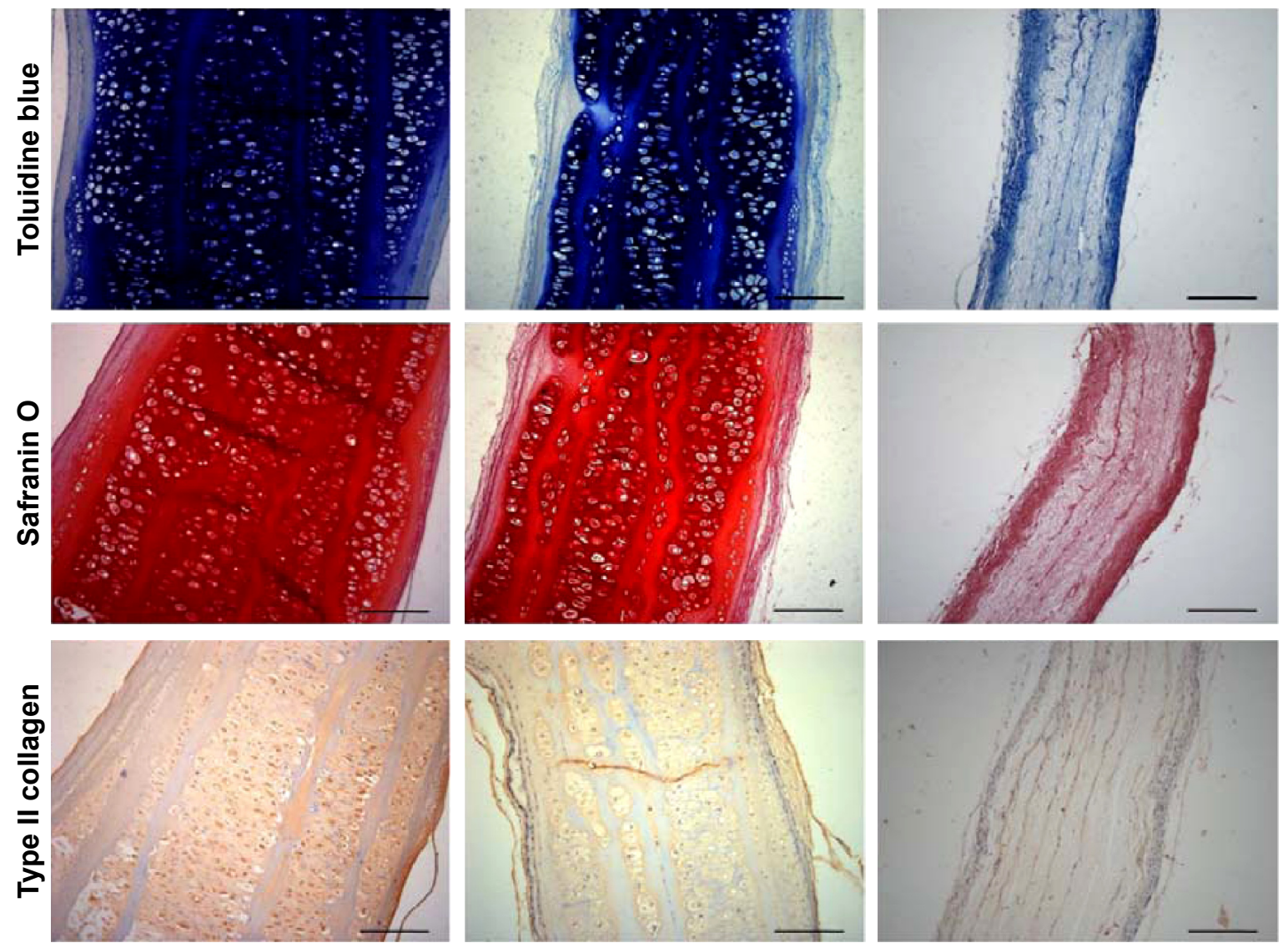

Chondrocytes group

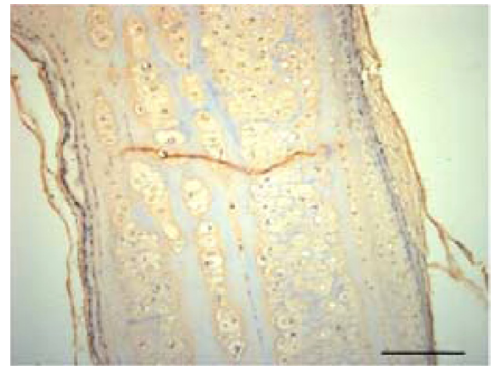

Coculture group

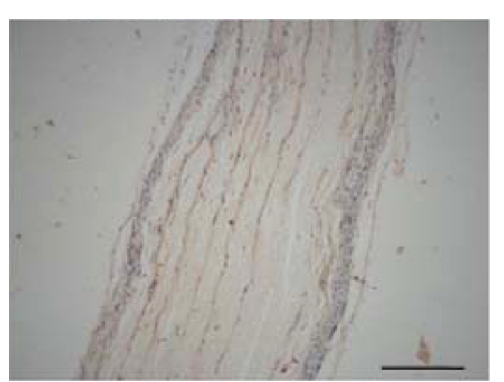

BMSCs group

Figure 5 Special histological staining with Toluidine blue and Safranin $\mathrm{O}$ and immunohistochemistry staining with type II collagen for cell-scaffold constructs in the different groups.

Note: Scale bars: $200 \mu \mathrm{m}$.

Abbreviation: BMSC, bone marrow stromal cell. 

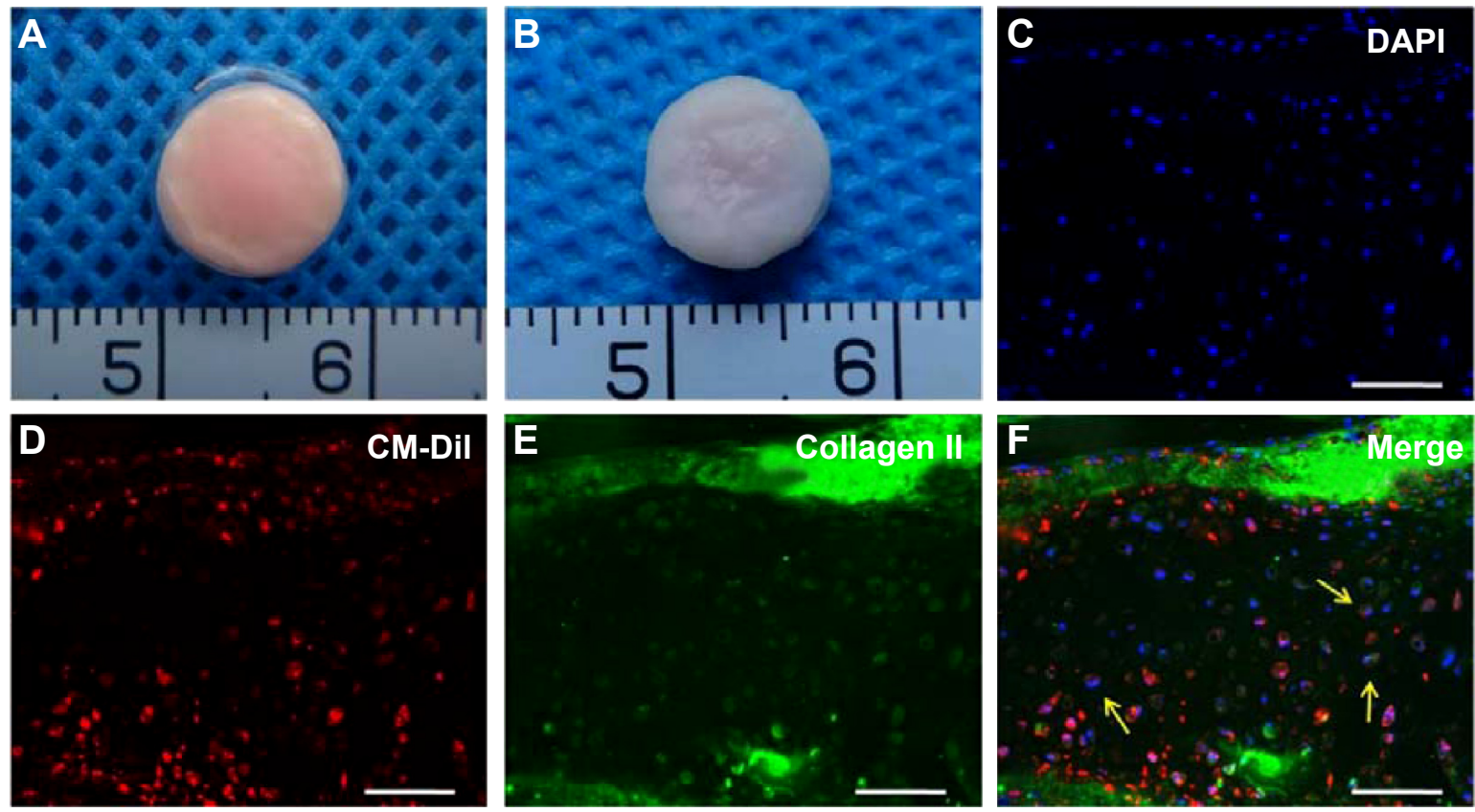

Figure 6 Labeling and tracing chondrogenesis of BMSCs.

Notes: (A) Gross view of cell-scaffold constructs formed by GT/PCL membranes seeded with coculture CM-Dil-labeled BMSCs and chondrocytes in vitro. (B) Gross view of cell-scaffold constructs after 12 weeks of implantation in the coculture group. (C-F) Immunofluorescence staining with type II collagen. Arrows show CM-Dil-labeled BMSCs overlapped with the type II collagen-positive cells in the constructs. Scale bars: $200 \mu \mathrm{m}$.

Abbreviations: DAPI, 4',6-diamidino-2-phenylindole; BMSC, bone marrow stromal cell; GT/PCL, gelatin/polycaprolactone.

in a red appearance also in the constructs. Moreover, a considerable number of CM-Dil-labeled BMSCs were still observed in the constructs even 12 weeks postimplantation, indicating that the CM-Dil dye could exist for a long time in vivo (Figure 6B). Furthermore, immunofluorescence staining demonstrated that most CM-Dil-labeled regions formed homogeneous cartilage-like tissue with typical lacuna structures and strong expression of type II collagen (Figure 6C-F), which confirmed that the BMSCs had been transformed into chondrocyte-like cells by chondrocytes during the coculture system and directly participated in the formation of cartilage.

\section{Discussion}

In this study, we have demonstrated the potential of GT/PCL nanofibrous membranes combined with a coculture of BMSCs and chondrocytes for application in cartilage engineering. Electrospun GT/PCL membranes could better mimic natural ECM and provide good cell affinity for both BMSC and chondrocyte adhesion and proliferation. Through engineering cartilage using GT/PCL membranes seeded with $\mathrm{BMSC} / \mathrm{chondrocytes}$ cocultures in a sandwich model in vitro and then implanting the constructs subcutaneously into nude mice for 12 weeks, the constructs showed well-distributed neocartilage tissue with an ivory-white appearance and good flexibility. The results indicate that BMSCs in the coculture group play an equal role compared with the chondrocyte group in the formation of cartilage.

Scaffolds play a vital role for engineering tissue and provide an environment for increasing the viability of cells and promoting synthesis of the neo-ECM. ${ }^{36}$ The choice of appropriate scaffold is critical for the quality of engineered cartilage. For scaffolds to be successfully used in tissue engineering, one essential property is that they should have good affinity for favoring cell adhesion. ${ }^{37}$ Recently, electrospinning technology, which can produce nanofibrous scaffolds with a high surface-area-to-volume ratio and fibers resembling the nanofibrous features of natural ECM with sufficient pores, has been shown to be beneficial in terms of cell adhesion, proliferation, and differentiation. ${ }^{11,38}$ Moreover, various synthetic and natural materials can be blended into nanocomposite electrospun scaffolds, observably improving cell-scaffold interactions for tissue engineering applications. ${ }^{16-18}$

The GT/PCL membrane is a representative nanocomposite electrospun scaffold blend composed of the natural material gelatin and the synthetic polymer PCL, and has been widely used for engineering diverse tissues, including nerve, bone, skin, cardiovascular, and cartilage. . $^{16,20,39-41}$ Our previous studies have further modified the fabrication of GT/PCL membranes to obtain finer and compositionally homogeneous hybrid nanofibers, and have demonstrated that 
the membranes could facilitate chondrocyte proliferation and promote cartilage regeneration. ${ }^{19,20}$

Since stem cell therapy shows greater potential for tissue regeneration, stem cells, especially BMSCs, are more likely to be used in future clinical applications. BMSCs can easily be obtained, and have a self-renewal capacity along with multiple differentiation potentials. ${ }^{22,23}$ Recent studies have indicated the differentiation potential of BMSCs for tissue regeneration on nanofibrous scaffolds. ${ }^{38,42}$ In this study, we also further investigated the affinity of GT/PCL membranes for BMSCs and, when combined with BMSCs, whether they could also generate neocartilage in the sandwich model. After seeding cells on a polymeric scaffold, cell adhesion and proliferation were the first cellular events to happen, and differentiation followed only after the cells were securely attached..$^{21}$ Based on SEM results, GT/PCL membranes had good cytocompatibility for both BMSCs and chondrocytes. This was partially due to the presence of a natural material, gelatin, in the nanocomposite GT/PCL membranes. Additionally, no cytotoxicity was observed when those cells were seeded on the membranes and cultured in vitro. These results indicate that GT/PCL membranes may also be an ideal scaffold for stem cell-based cartilage engineering.

Nanofibrous scaffolds combined with BMSCs may provide an attractive therapeutic strategy for the clinical applicability of repairing cartilage defects. However, it still remains a challenge to reproduce the functional properties of native cartilage while using only BMSCs..$^{43}$ Moreover, the current BMSC-based cartilage regeneration tended toward hypertrophy and calcification in the presence of growth factors, especially at heterotopic sites. ${ }^{26,44}$ Therefore, further optimization of the induction mode of BMSCs must be performed in order to reach levels similar to those produced by chondrocytes.

Recent studies have indicated that paracrine soluble factors, such as TGF- $\beta 1$, IGF-1, and BMP-2, released by chondrocytes are able to induce the chondrogenesis of BMSCs in a coculture system. ${ }^{29,30}$ Furthermore, the coculture system could inhibit the terminal differentiation and hypertrophy of BMSCs, resulting in stable cartilage formation. ${ }^{27,45}$ However, very few studies focus on the chondrogenesis of BMSCs by chondrocytes in a coculture system on electrospun nanofibrous substrates.

In this study, we used a coculture strategy instead of pure chondrocytes to engineer cartilage using GT/PCL membranes seeded with BMSC/chondrocyte cocultures in the sandwich model in vitro and then implanting the constructs subcutaneously into nude mice for 12 weeks to evaluate the formation of cartilaginous tissue. The constructs had not formed any new tissues, with almost no mechanical properties, in the BMSC group. As compared with the constructs in the BMSC group, all constructs in the coculture group formed cartilage-like tissue with an ivory-white appearance and showed significantly greater thickness, wet weight, GAG content, and improved mechanical properties, with no statistically significant difference compared with the chondrocyte group. Our experimental data suggest that pure BMSCs could not form new tissues without the induction of chondrogenic factors. However, the GT/PCL membranes could facilitate the formation of cartilage-like tissue in vivo by the coculture system, which was further supported by subsequent histological and immunohistochemical observations. These findings indicate that BMSCs played a role equivalent to the chondrocytes in the chondrocyte group in the formation of cartilage. Moreover, the chondrogenic differentiation of BMSCs was further proved by tracing CM-Dil-labeled BMSCs in vivo for the expression of type II collagen from immunofluorescence staining. BMSCs could directly participate in the formation of cartilage when on the GT/PCL membranes using the coculture strategy. Additionally, we also engineered constructs with GT/PCL membranes seeded with pure BMSCs in the presence of TGF- $\beta 3$ for 4 weeks' induction in vitro, and then implanted the constructs into nude mice for 12 weeks. Unfortunately, the constructs did not form effective cartilage-like tissues, with little cartilage diffused distribution in the constructs (data not shown). We considered that the reason for this may be that the growth factor had poor induction efficiency, despite the lengthy induction in vitro, and was not able to fully penetrate into the interior of the constructs in the sandwich model. However, BMSCs could form high-quality and well-distributed neocartilage in the presence of low numbers of chondrocytes using the aforementioned coculture strategy. Therefore, the coculture strategy with electrospun nanofibrous scaffolds is an easy, economical, effective, and potentially clinically feasible approach for cartilage repair, since a small number of chondrocytes could be harvested from healthy tissue without obvious damage, avoiding the in vitro massive expansion and corresponding issues related to dedifferentiation.

Besides cytocompatibility, the appropriate biodegradation of scaffolds also plays a critical role in the application of tissue engineering, since the degradation behavior of scaffolds also can affect cell growth and subsequent tissue regeneration..$^{46,47}$ Thus, the ideal biodegradation rate of the GT/PCL membranes needs to be controlled to match the rate of cartilage regeneration. In the present study, we used a 50:50 ratio of gelatin and PCL for the GT/PCL 
membranes, and each GT/PCL membrane layer was most detectable after 12 weeks of implantation, indicating that the degradation rate of our electrospun GT/PCL membranes is somewhat slow. However, the degradation rate of GT/PCL membranes can also be readily controlled by adjusting the ratio of its two components, gelatin and PCL, since gelatin degrades rapidly both in vitro and in vivo, affecting the degradation process as well as the mechanical properties. Recent research has demonstrated that GT/PCL 70:30 might be a relatively suitable ratio for 3-D cartilage regeneration, especially for cartilage with a complicated shape. ${ }^{48}$ This new finding indicates that the degradation rate of GT/PCL membranes should also be precisely controlled for stem cell-based cartilage engineering with complicated shapes, such as human ear-shaped cartilage and tracheal cartilage, in future studies.

\section{Conclusion}

In summary, this study further broke the limitations of chondrocytes in our previous studies, and demonstrated that electrospun GT/PCL membranes facilitated the formation of high-quality and well-distributed neocartilage using a BMSC/chondrocyte coculture strategy, indicating that GT/PCL membranes were also a suitable scaffold for stem cell-based cartilage engineering. Furthermore, electrospun nanofibrous scaffolds, combined with stem cell coculture strategy, might also provide a potentially clinically feasible approach for cartilage repairs.

\section{Acknowledgments}

This study was supported by the National Natural Science Fund of China (31370982, 31200735, 81271726, 81370117), Key Projects of Shanghai Education Committee (14ZZ113), Shanghai Natural Science Fund for Youth Scholars (12ZR1446500), Science and Technology Development Fund of Shanghai Pudong (PKJ2012-Y48), Shanghai Municipal Commission of Health and Family Planning (20144Y0166), the Biomedical Engineering fund of Shanghai Jiao Tong University (YG2012MS36, YG2012MS35), the College Young Teachers' Training and Funding Project of Shanghai (ZZjdyx12117, ZZjdyx12124), and the College Young Teachers' Training and Funding Project of Shanghai Jiao Tong University School of Medicine.

\section{Disclosure}

No competing financial interests exist. The authors report no conflicts of interest in this work.

\section{References}

1. Vacanti CA, Paige KT, Kim WS, Sakata J, Upton J, Vacanti JP. Experimental tracheal replacement using tissue-engineered cartilage. J Pediatr Surg. 1994;29(2):201-204; discussion 204-205.

2. Wu W, Cheng X, Zhao Y, Chen F, Feng X, Mao T. Tissue engineering of trachea-like cartilage grafts by using chondrocyte macroaggregate: experimental study in rabbits. Artif Organs. 2007;31(11):826-834.

3. Bermueller C, Schwarz S, Elsaesser AF, et al. Marine collagen scaffolds for nasal cartilage repair: prevention of nasal septal perforations in a new orthotopic rat model using tissue engineering techniques. Tissue Eng Part A. 2013;19(19-20):2201-2214.

4. Barnewitz D, Endres M, Kruger I, et al. Treatment of articular cartilage defects in horses with polymer-based cartilage tissue engineering grafts. Biomaterials. 2006;27(14):2882-2889.

5. Liu Y, Zhang L, Zhou G, et al. In vitro engineering of human earshaped cartilage assisted with CAD/CAM technology. Biomaterials. 2010;31(8):2176-2183.

6. Thissen H, Chang KY, Tebb TA, et al. Synthetic biodegradable microparticles for articular cartilage tissue engineering. J Biomed Mater Res A. 2006;77(3):590-598.

7. Liu X, Holzwarth JM, Ma PX. Functionalized synthetic biodegradable polymer scaffolds for tissue engineering. Macromol Biosci. 2012;12(7):911-919.

8. Dvir T, Timko BP, Kohane DS, Langer R. Nanotechnological strategies for engineering complex tissues. Nat Nanotechnol. 2011;6(1): $13-22$.

9. Jin L, Wang T, Zhu ML, et al. Electrospun fibers and tissue engineering. J Biomed Nanotechnol. 2012;8(1):1-9.

10. Alamein MA, Stephens S, Liu Q, Skabo S, Warnke PH. Mass production of nanofibrous extracellular matrix with controlled 3D morphology for large-scale soft tissue regeneration. Tissue Eng Part C Methods. 2013;19(6):458-472.

11. Chen ZG, Wang PW, Wei B, Mo XM, Cui FZ. Electrospun collagenchitosan nanofiber: a biomimetic extracellular matrix for endothelial cell and smooth muscle cell. Acta Biomater. 2010;6(2):372-382.

12. Hajiali H, Shahgasempour S, Naimi-Jamal MR, Peirovi H. Electrospun PGA/gelatin nanofibrous scaffolds and their potential application in vascular tissue engineering. Int $J$ Nanomedicine. 2011;6: 2133-2141.

13. Alves da Silva ML, Martins A, Costa-Pinto AR, et al. Cartilage tissue engineering using electrospun PCL nanofiber meshes and MSCs. Biomacromolecules. 2010;11(12):3228-3236.

14. Hoveizi E, Nabiuni M, Parivar K, Rajabi-Zeleti S, Tavakol S. Functionalisation and surface modification of electrospun polylactic acid scaffold for tissue engineering. Cell Biol Int. 2014;38(1):41-49.

15. Shtrichman R, Zeevi-Levin N, Zaid R, et al. The generation of hybrid electrospun nanofiber layer with extracellular matrix derived from human pluripotent stem cells, for regenerative medicine applications. Tissue Eng Part A. 2014;20(19-20):2756-2767.

16. Ghasemi-Mobarakeh L, Prabhakaran MP, Morshed M, Nasr-Esfahani MH, Ramakrishna S. Electrospun poly(epsilon-caprolactone)/gelatin nanofibrous scaffolds for nerve tissue engineering. Biomaterials. 2008;29(34):4532-4539.

17. Han J, Lazarovici P, Pomerantz C, Chen X, Wei Y, Lelkes PI. Co-electrospun blends of PLGA, gelatin, and elastin as potential nonthrombogenic scaffolds for vascular tissue engineering. Biomacromolecules. 2011;12(2):399-408.

18. He X, Fu W, Feng B, et al. Electrospun collagen-poly(L-lactic acidco- $\varepsilon$-caprolactone) membranes for cartilage tissue engineering. Regen Med. 2013;8(4):425-436.

19. Feng B, Tu H, Yuan H, Peng H, Zhang Y. Acetic-acid-mediated miscibility toward electrospinning homogeneous composite nanofibers of GT/PCL. Biomacromolecules. 2012;13(12):3917-3925.

20. Xue J, Feng B, Zheng R, et al. Engineering ear-shaped cartilage using electrospun fibrous membranes of gelatin/polycaprolactone. Biomaterials. 2013;34(11):2624-2631. 
21. Homicz MR, Schumacher BL, Sah RL, Watson D. Effects of serial expansion of septal chondrocytes on tissue-engineered neocartilage composition. Otolaryngol Head Neck Surg. 2002;127(5):398-408.

22. Pittenger MF, Mackay AM, Beck SC, et al. Multilineage potential of adult human mesenchymal stem cells. Science. 1999;284(5411):143-147.

23. Barry FP, Murphy JM. Mesenchymal stem cells: clinical applications and biological characterization. Int J Biochem Cell Biol. 2004;36(4):568-584.

24. Fuchs JR, Hannouche D, Terada S, Vacanti JP, Fauza DO. Fetal tracheal augmentation with cartilage engineered from bone marrow-derived mesenchymal progenitor cells. J Pediatr Surg. 2003;38(6):984-987.

25. He X, Fu W, Zheng J. Cell sources for trachea tissue engineering: past, present and future. Regen Med. 2012;7(6):851-863.

26. Pelttari K, Winter A, Steck E, et al. Premature induction of hypertrophy during in vitro chondrogenesis of human mesenchymal stem cells correlates with calcification and vascular invasion after ectopic transplantation in SCID mice. Arthritis Rheum. 2006;54(10):3254-3266.

27. Bian L, Zhai DY, Mauck RL, Burdick JA. Coculture of human mesenchymal stem cells and articular chondrocytes reduces hypertrophy and enhances functional properties of engineered cartilage. Tissue Eng Part A. 2011;17(7-8):1137-1145.

28. Kang N, Liu X, Guan Y, et al. Effects of co-culturing BMSCs and auricular chondrocytes on the elastic modulus and hypertrophy of tissue engineered cartilage. Biomaterials. 2012;33(18):4535-4544.

29. Liu X, Sun H, Yan D, et al. In vivo ectopic chondrogenesis of BMSCs directed by mature chondrocytes. Biomaterials. 2010;31(36): 9406-9414.

30. Fischer J, Dickhut A, Rickert M, Richter W. Human articular chondrocytes secrete parathyroid hormone-related protein and inhibit hypertrophy of mesenchymal stem cells in coculture during chondrogenesis. Arthritis Rheum. 2010;62(9):2696-2706.

31. Xue JX, Gong YY, Zhou GD, Liu W, Cao Y, Zhang WJ. Chondrogenic differentiation of bone marrow-derived mesenchymal stem cells induced by acellular cartilage sheets. Biomaterials. 2012;33(24):5832-5840.

32. Gong YY, Xue JX, Zhang WJ, Zhou GD, Liu W, Cao Y. A sandwich model for engineering cartilage with acellular cartilage sheets and chondrocytes. Biomaterials. 2011;32(9):2265-2273.

33. Hoemann CD. Molecular and biochemical assays of cartilage components. Methods Mol Med. 2004;101:127-156.

34. Yan D, Zhou G, Zhou X, et al. The impact of low levels of collagen IX and pyridinoline on the mechanical properties of in vitro engineered cartilage. Biomaterials. 2009;30(5):814-821.

35. Zong C, Xue D, Yuan W, et al. Reconstruction of rat calvarial defects with human mesenchymal stem cells and osteoblast-like cells in polylactic-co-glycolic acid scaffolds. Eur Cell Mater. 2010;20:109-120.
36. Carletti E, Motta A, Migliaresi C. Scaffolds for tissue engineering and 3D cell culture. Methods Mol Biol. 2011;695:17-39.

37. Lutolf MP, Hubbell JA. Synthetic biomaterials as instructive extracellular microenvironments for morphogenesis in tissue engineering. Nat Biotechnol. 2005;23(1):47-55.

38. Prabhakaran MP, Venugopal JR, Ramakrishna S. Mesenchymal stem cell differentiation to neuronal cells on electrospun nanofibrous substrates for nerve tissue engineering. Biomaterials. 2009;30(28):4996-5003.

39. Alvarez Perez MA, Guarino V, Cirillo V, Ambrosio L. In vitro mineralization and bone osteogenesis in poly( $\varepsilon$-caprolactone)/gelatin nanofibers. J Biomed Mater Res A. 2012;100(11):3008-3019.

40. Tigli RS, Kazaroglu NM, Mav ISB, Gumusderel IOM. Cellular behavior on epidermal growth factor (EGF)-immobilized PCL/gelatin nanofibrous scaffolds. J Biomater Sci Polym Ed. Epub June 16, 2010.

41. Heydarkhan-Hagvall S, Schenke-Layland K, Dhanasopon AP, et al. Three-dimensional electrospun ECM-based hybrid scaffolds for cardiovascular tissue engineering. Biomaterials. 2008;29(19):2907-2914.

42. Xin X, Hussain M, Mao JJ. Continuing differentiation of human mesenchymal stem cells and induced chondrogenic and osteogenic lineages in electrospun PLGA nanofiber scaffold. Biomaterials. 2007;28(2):316-325.

43. Mauck RL, Yuan X, Tuan RS. Chondrogenic differentiation and functional maturation of bovine mesenchymal stem cells in long-term agarose culture. Osteoarthritis Cartilage. 2006;14(2):179-189.

44. Yang Z, Zou Y, Guo XM, et al. Temporal activation of $\beta$-catenin signaling in the chondrogenic process of mesenchymal stem cells affects the phenotype of the cartilage generated. Stem Cells Dev. 2012;21(11):1966-1976.

45. Cui X, Hasegawa A, Lotz M, D'Lima D. Structured three-dimensional co-culture of mesenchymal stem cells with meniscus cells promotes meniscal phenotype without hypertrophy. Biotechnol Bioeng. 2012;109(9):2369-2380.

46. Sung HJ, Meredith C, Johnson C, Galis ZS. The effect of scaffold degradation rate on three-dimensional cell growth and angiogenesis. Biomaterials. 2004;25(26):5735-5742.

47. Dong Y, Yong T, Liao S, Chan CK, Stevens MM, Ramakrishna S. Distinctive degradation behaviors of electrospun polyglycolide poly(DL-lactide-co-glycolide), and poly(L-lactide-co-epsilon-caprolactone) nanofibers cultured with/without porcine smooth muscle cells. Tissue Eng Part A. 2010;16(1):283-298.

48. Zheng R, Duan H, Xue J, et al. The influence of gelatin/PCL ratio and 3-D construct shape of electrospun membranes on cartilage regeneration. Biomaterials. 2014;35(1):152-164.
International Journal of Nanomedicine

\section{Publish your work in this journal}

The International Journal of Nanomedicine is an international, peerreviewed journal focusing on the application of nanotechnology in diagnostics, therapeutics, and drug delivery systems throughout the biomedical field. This journal is indexed on PubMed Central, MedLine, CAS, SciSearch $®$, Current Contents $\AA /$ Clinical Medicine,

\section{Dovepress}

Journal Citation Reports/Science Edition, EMBase, Scopus and the Elsevier Bibliographic databases. The manuscript management system is completely online and includes a very quick and fair peer-review system, which is all easy to use. Visit http://www.dovepress.com/ testimonials.php to read real quotes from published authors. 